\title{
Linx
}

Revue des linguistes de l'université Paris X Nanterre

$12 \mid 2002$

"Comme la lettre dit la vie »

\section{Co-énonciation et expression du sujet de l'impératif en ancien français}

\section{Evelyne Oppermann}

\section{(2) OpenEdition}

1 Journals

Édition électronique

URL : https://journals.openedition.org/linx/1306

DOI : 10.4000/linx.1306

ISSN : 2118-9692

\section{Éditeur}

Presses universitaires de Paris Nanterre

\section{Édition imprimée}

Date de publication : 1 octobre 2002

Pagination : 209-216

ISSN : 0246-8743

\section{Référence électronique}

Evelyne Oppermann, « Co-énonciation et expression du sujet de l'impératif en ancien français », Linx

[En ligne], 12 | 2002, mis en ligne le 10 octobre 2012, consulté le 28 juin 2022. URL : http:// journals.openedition.org/linx/1306 ; DOI : https://doi.org/10.4000/linx.1306

Ce document a été généré automatiquement le 29 septembre 2020.

Département de Sciences du langage, Université Paris Ouest 


\title{
Co-énonciation et expression du sujet de l'impératif en ancien français
}

\author{
Evelyne Oppermann
}

\section{Introduction}

1 En français contemporain, le verbe à l'impératif se caractérise par le fait qu'il s'avère incompatible avec l'expression de son sujet. Selon l'analyse de Danon-Boileau, Morel et Perrin (1992), cette propriété morpho-syntaxique s'expliquerait par le statut énonciatif particulier de l'énoncé impératif et plus précisément par le fait que ce type d'énoncé s'inscrit dans la co-locution, sans être fondé sur la co-énonciation : il implique la co-présence du locuteur et de l'allocutaire, mais le locuteur n'y situe pas ses paroles par rapport à l'attitude et aux pensées de son allocutaire ${ }^{1}$.

2 L'impératif de l'ancien français se révèle, en revanche, parfois compatible avec l'expression de son sujet $t u / v o s$ :

Avec l'impératif le français moderne n'exprime pas le sujet ; c'est même ce qui pour nous donne à ce mode sa physionomie particulière, le distingue de l'indicatif par exemple. Il n'en est pas de même en ancien français, où à l'occasion on n'éprouve aucune gêne à faire précéder l'impératif du pronom sujet :... (Foulet, rééd. 1990 : 215)

Les deux occurrences suivantes comportent ainsi une forme tu/vos qui peut être analysée, d'un point de vue syntaxique, comme le sujet de la phrase impérative ${ }^{2}$ :

\begin{tabular}{|c|c|}
\hline (1) & Jel <je le> te di et tu l'entens. (Aucassin, XV/9-15) \\
\hline 2) & Sire, vos me soiez pastres et deffenderres et conduisierres,... (Queste del st Graal, p. 96/11-12) \\
\hline
\end{tabular}



l'aide de deux critères : «Le pronom sujet accompagne quelquefois le verbe à l'impératif, soit pour des raisons d'expressivité, soit pour des raisons rythmiques » (Hasenohr, 1990, § 86), notamment "pour éviter qu'une forme atone apparaisse en tête de phrase » (Ménard, 1988, § 57). Si (1) et (2) rendent bien compte au moins de ce deuxième critère, une telle analyse soulève cependant aussi des interrogations.

Tout d'abord, comment peut-on différencier les énoncés exprimant le sujet de l'impératif comme (1) et (2) des énoncés impératifs (3) et (4), où c'est un adverbe - or, car - qui permet à un pronom régime atone de rester en position proclitique, tout en constituant aussi la marque d'une certaine expressivité ${ }^{3}$ ?

(3) Or me dites < dites-moi donc>, conuissiez vos / le roi Urïen? (Conte du Graal, v. 7896-97)

(4) Va, car me $\underline{\text { di }}<$ dis-moi donc> / se tu es boene chose ou non. (Chev. au lion, v. 326-27)

n remarque par ailleurs que les explications avancées s'appliquent, dans les manuels, régulièrement à des occurrences dans lesquelles le pronom sujet est antéposé au verbe. Que faire alors d'un énoncé comme (5), où l'emploi de vus ne peut s'expliquer par le critère syntaxique évoqué plus haut (vu sa place derrière le verbe) et ne semble pas non plus correspondre à une marque d'insistance particulière?

(5) Si veirement cum ceo feis, / si aiez vus de mei merci. (Gormont, v. 647-48)

Ceci nous laisse penser que les deux critères retenus - l'ordre des mots et l'expressivité ne mettent pas en évidence la spécificité des tournures impératives avec sujet en ancien français et que la prise en considération d'autres concepts, notamment de ceux de colocution et de co-énonciation, permettrait de la cerner davantage.

Si l'impératif est généralement employé sans sujet en français à cause de son ancrage dans la co-locution, nous faisons ainsi l'hypothèse qu'il devient, en ancien français, compatible avec l'expression du sujet dès qu'il ne s'inscrit pas exclusivement dans la colocution, mais implique de plus la présence d'une relation co-énonciative entre le locuteur-énonciateur et son allocutaire, qui devient alors co-énonciateur. C'est ce que nous essaierons de montrer à partir d'une analyse des différents types de cotextes ${ }^{4}$ dans lesquels figurent les énoncés à l'impératif ${ }^{5}$ - désormais E(imp) - avec sujet pronominal de notre corpus ${ }^{6}$, que ce sujet soit anté- ou postposé à la forme verbale.

\section{Le sujet est antéposé au verbe}

Les énoncés dans lesquels le sujet de l'impératif est exprimé devant la forme verbale apparaissent dans plusieurs types de cotextes. A l'intérieur des E(imp) relevés, une première distinction peut être établie en fonction des propriétés de leur cotexte gauche, celui-ci pouvant être attribué au même locuteur que E(imp) ou à un locuteur différent. 


\section{A. E(imp) et son cotexte gauche sont attribués au même locuteur}

10 Lorsqu'un E(imp) avec sujet est énoncé par le même locuteur que son cotexte gauche, il est employé, à l'intérieur de notre corpus, essentiellement dans l'une des trois constructions suivantes :

- E(imp) est coordonné par et à un premier énoncé déclaratif :

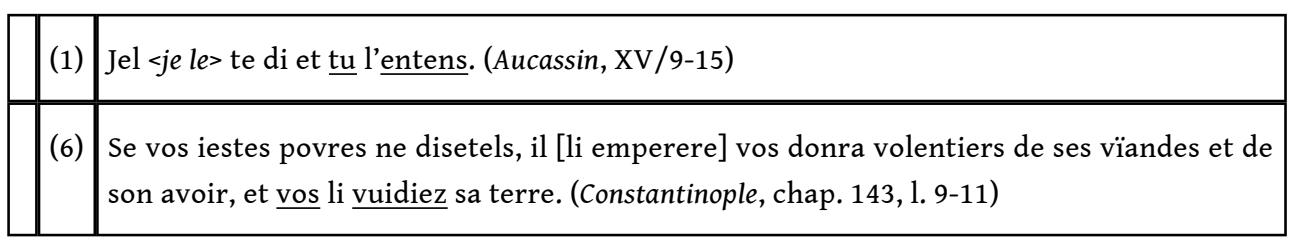

- E(imp) régit une proposition subordonnée hypothétique :

(7) Vos me sivrez a la feste seint Michel, / Si recevrez la lei <religion> de chrestïens, / Serez ses hom par honur e par ben. / S'en volt ostages, e vos l'en enveiez, / U dis u vint, pur lui afiancer. (Roland, v. 37-41)

- E(imp) est précédé d'une séquence phrastique introduite par un appellatif avec expansion :

(2) Biax douz peres, qui deistes en l'evangile de vos meismes : « Je sui bons pastres, et li bons pastres met s'ame por ses oeilles <brebis>, mes ce ne fet pas li marcheanz pastres; ançois let ses oeilles sanz garde tant que li leux <loup> les estrangle et devore si tost come il i vient "; Sire, vos me soiez pastres et deffenderres et conduisierres, si que je soie de vos oeilles. (Queste del st Graal, p. 96/7-13)

11 Bien qu'elles aient des propriétés syntaxiques fort différentes, ces occurrences ont un trait caractéristique en commun: E(imp) n'y constitue pas, en tant que tel, un énoncé autonome, « un isolat dans la trame discursive » (Danon-Boileau, Morel, Perrin, 1992: 161), contrairement à ce qui se produit en général pour les injonctions à l'impératif, et notamment pour celles qui sont introduites par un adverbe - cf. (3) et (4). Nos impératifs avec sujet sont en effet directement articulés à leur cotexte gauche ; et celui-ci permet, dans les trois cas de figure, de poser l'existence d'une relation co-énonciative. Ceci est particulièrement évident dans (1) et (6), où le cotexte gauche de E(imp) est constitué d'un énoncé déclaratif. Or,

la modalité qui affecte un énoncé de forme déclarative est ontique. L'énonciateur situe l'opinion qu'il énonce ou le phénomène dont il pose l'existence par rapport à la pensée qu'il prête au co-énonciateur. Il définit un état de co-énonciation qui oscille entre trois valeurs : consensualité, discordance, 'objectivité'. (ibid., p. 158).

Une telle coordination semble d'ailleurs peu probable en français moderne, où E(imp) serait alors remplacé par une injonction indirecte prenant la forme d'un énoncé déclaratif, notamment au futur: Il vous donnera volontiers de ses vivres et de ses richesses, et vous quitterez sa terre (6)

Dans (7), E(imp) ne représente pas non plus un énoncé autonome : l'injonction n'est pas formulée de manière absolue, mais elle est liée à une condition énoncée au préalable, ce 
lien avec le cotexte immédiat étant d'ailleurs souligné par l'emploi d'un et « de reprise ${ }^{8}$ en début de E(imp) ainsi que par le pronom anaphorique en (qui représente le substantif ostages). Et même si ce cotexte prend la forme d'une subordonnée hypothétique, il relève néanmoins d'une modalité ontique : le locuteur-énonciateur y présente un état de choses comme possible, ce que souligne l'emploi du présent de l'indicatif volt (qui est ici une graphie pour vuelt). Notons par ailleurs que les injonctions de (7) qui précèdent directement $\mathrm{E}(\mathrm{imp})$ prennent la forme d'énoncés au futur, qui impliquent donc, en tant qu'énoncés déclaratifs, également une relation co-énonciative.

Enfin, l'occurrence (2) révèle, elle aussi, la présence d'un état de co-énonciation. Une comparaison avec l'extrait suivant met en évidence ce trait :

(8) Vaslez, fet il, tu qui la viens, / qui le costel an ta main tiens, / mostrez moi quex est li rois. (Conte du Graal, v. 915-17)

Dans (8) comme dans (2), le cotexte gauche de E(imp) comporte un appellatif avec une expansion apportant des précisions concernant la personne de l'allocutaire; mais cette expansion n'a pas la même fonction dans les deux cas. Ainsi, les indications fournies par les propositions relatives de (8) semblent surtout servir à identifier l'allocutaire de l'énoncé ; elles correspondent, plus précisément, à des « propriétés discriminantes » dans une situation d'énonciation donnée ${ }^{9}$, grâce auxquelles le jeune homme concerné peut s'identifier sans difficulté, parmi les autres personnes présentes, comme étant l'allocutaire des paroles prononcées. De ce fait, la présence de cette expansion se justifie dans le cadre de la co-locution: elle permet au locuteur de préciser cette relation et d'éviter toute ambiguïté ou confusion la concernant. Il n'est alors pas étonnant que l'impératif mostrez soit employé sans sujet. L'exemple (2) témoigne en revanche d'un cas de figure différent. Dans cette demande que le locuteur Perceval adresse à Dieu, il n'y a pas d'ambiguïté au plan co-locutoire. Les informations données dans la relative, avec référence à l'Evangile, mettent alors surtout en évidence le désir du locuteur-énonciateur de définir un état de co-énonciation avec son co-énonciateur : elles n'évoquent pas des propriétés pouvant être discriminantes dans la situation d'énonciation rapportée, mais apportent plutôt une caractérisation de l'allocutaire, à travers laquelle Perceval cherche aussi à se présenter comme un bon chrétien, qui connaît l'Ecriture Sainte, et à souligner ainsi ses convictions et sa foi. L'on peut rattacher à ce type d'emploi l'occurrence suivante, qui représente le plus ancien exemple d'impératif avec sujet de notre corpus.

(9) O Deus, vers rex, Jesu Crist, / ci <qui> tal don fais per ta mercit, / chi <qui> per huna <une > confession / vide perdones al ladrun, / nos te laudam et noit et di, / de nos aies vera mercit; / tu nos perdone celz pecaz / q'e nos vetdest tua pietad. <les péchés que ta miséricorde verrait en nous>. (Passion, v. 301-08)

Comme pour (1), (6) et (7), le cotexte gauche de E(imp) met en place ici un état de coénonciation; il permet au locuteur de poser des faits dont il pense qu'ils conviennent à son allocutaire, ce qui rend possible l'expression du sujet de l'impératif. 


\section{B. E(imp) et son cotexte gauche sont attribués à des locuteurs différents}

Nous pouvons faire un constat comparable lorsque l'impératif avec sujet et son cotexte gauche sont attribués à des locuteurs distincts. Contrairement aux occurrences de A., E (imp) y constitue alors une intervention autonome, mais son énonciation s'explique directement par celle de l'intervention précédente avec laquelle il forme un échange verbal. Ce lien entre $\mathrm{E}(\mathrm{imp})$ et son cotexte gauche est d'ailleurs indiqué, dans nos exemples, par l'emploi d'une conjonction - mais ou et - en tête de la séquence <sujet + impératif > :

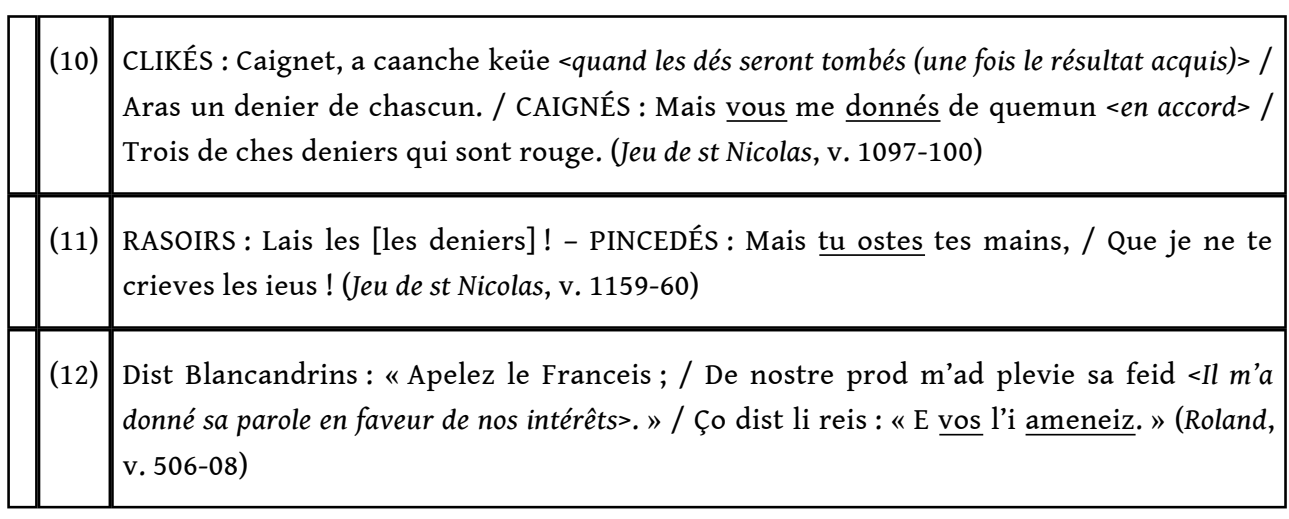

Dans ces trois occurrences, l'impératif avec sujet, tout en représentant une intervention initiative ${ }^{10}$, qui suscite une réaction de la part de son allocutaire, est en même temps une intervention réactive qui « répond " à un premier énoncé (qui est souvent également injonctif). Il est ainsi formulé en fonction de ce que le locuteur sait déjà, grâce à ce cotexte gauche, de l'attitude et de la volonté de son allocutaire et ne peut donc pas s'inscrire exclusivement dans la co-locution: il y a aussi définition d'un état de coénonciation, dans la mesure où le locuteur de $\mathrm{E}(\mathrm{imp})$ situe bien cette intervention par rapport aux pensées et désirs de son allocutaire, soit pour s'en démarquer, soit pour les approuver. Dans (10) et (11), <Mais + $\mathrm{E}(\mathrm{imp})>$ témoigne d'un désaccord entre les interlocuteurs, celui-ci portant soit sur l'action exprimée par le verbe de la première intervention ou un aspect de celle-ci [cf. (10) où donner " a caanche keüe " s'oppose à donner "de quemun »], soit sur l'agent de cette action [cf. (11) où Pincedé part du principe que ce n'est pas lui, mais son allocutaire, qui doit lâcher les deniers]. Dans les deux cas, le locuteur s'oppose directement aux prétentions ${ }^{11}$ que comporte l'énoncé déclaratif ou impératif qui précède et essaie de modifier l'attitude de son allocutaire. A ce titre, on peut dire que ces deux énoncés traduisent un "forçage de consensualité co-énonciative» (Danon-Boileau, Morel, Perrin, op. cit., p. 162). Dans ce type de cotexte, l'impératif avec sujet cède d'ailleurs souvent la place à un énoncé de forme déclarative, en particulier à une injonction au futur :

(13) LI TAVRENIERS : Paie denier, et a l'autre eure / Aras le pinte pour maaille. / C'est a douze deniers, sans faille : / Paie un denier ou boi encore. / AUBERONS : Mais le maille prenderés ore / Et au revenir le denier ! (Jeu de st Nicolas, v. 274-79) 

forme verbale. Dans ces occurrences, nous verrons que l'expression du sujet pronominal peut s'expliquer, comme pour les énoncés traités en 1., par la présence d'un état de coénonciation (qu'il est en général possible de reconstruire à l'aide du cotexte gauche de $\mathrm{E}$ (imp)). Deux classes peuvent être distinguées à l'intérieur de ces énoncés - les E(imp) positifs et les $\mathrm{E}(\mathrm{imp})$ négatifs ${ }^{12}$. Leur cotexte gauche est, dans les deux cas, toujours attribué au même locuteur que l'impératif.

\section{A. E(imp) est un énoncé positif}

Lorsque tu/vos est le sujet d'un impératif positif, son cotexte gauche a des propriétés déjà relevées pour les impératifs avec sujet antéposé. Il comporte en effet régulièrement un appellatif complété par une expansion plus ou moins longue. Et cette expansion a une fonction comparable à celle relevée dans (2) et (9) :

(5) Aie ! pere Deu, dist il, / qui enz en la seinte cruiz fus mis, / a vendresdi mort i soffri, / dunt tut tun pople reinsis <rachetas $>$, / eu seinte sepulchre fustes mis, / e au tierz jor surrexis. / Si veirement cum ceo feis, / si aiez vus de mei merci. (Gormont, v. 641-48)

(14) Dieux ! fait elle, qui le revel <allégresse> / En l'umain lignage meÿs, / Quant char et sanc ou corps preïs / De la Vierge pucelle sage / Par noncion de ton message, / Puis en fuz nez com homs en terre, / Li troys roy te vindrent requerre / (...) / Puis fuz mors en la croiz et mis, / D'enfer gettas tous tes amis, / Et au tiers jour de passion / Venis a resurrection, / Es cieulx montas en joie clere, / Ou siez a la destre ton pere, / Les vifz et les mors jugeras, / Et a tous leur loyer rendras! / Dieux, si com c'est droite creance, / Octroyes tu par ta puissance / Qu'a femme ma fillole preigne / Le filz au conte de Bretaigne! (Galeran, v. 1880-902)

Comme (2) et (9), ces deux occurrences s'adressent à Dieu et l'appellatif nomme explicitement l'allocutaire. Les propositions qui suivent n'ont donc pas pour but d'identifier celui-ci, mais elles permettent au locuteur de définir une relation coénonciative : celui-ci y caractérise l'allocutaire à travers l'évocation de sa vie, tout en 
soulignant en même temps ses propres convictions religieuses. Ainsi, le début de ces interventions correspond à un véritable Credo et leur valeur assertive est mise en évidence - cf. Si veirement cum ceo feis (5) et si com c'est droite creance (14). Le locuteur y affirme de cette manière des faits dont il sait que son allocutaire les prend également en charge. Celui-ci est alors bien posé comme un véritable co-énonciateur, ce qui rend possible l'expression du sujet de l'impératif.

\section{B. E(imp) est un énoncé négatif}

Les $\mathrm{E}(\mathrm{imp})$ correspondant à des énoncés négatifs présentent un cas de figure différent, dans la mesure où ils ont d'entrée un statut particulier face à la co-énonciation. En effet, la négation implique forcément la présence d'un énonciateur et d'un co-énonciateur, même si elle porte sur un verbe conjugué à l'impératif :

le rôle du discordanciel ne est précisément de signaler l'existence d'un contenu de pensée consensuellement partagé, à partir de quoi l'énonciateur peut construire une discordance. (...) La mise en jeu de la négation rapproche ainsi l'impératif du déclaratif. (Danon-Boileau, Morel, Perrin, op. cit., p. 166)

Les E(imp) négatifs n'ont donc pas besoin d'un cotexte gauche immédiat particulier pour que la présence d'une relation de co-énonciation et l'emploi possible du sujet de l'impératif y soient justifiés. Cependant, la plupart de nos impératifs négatifs avec sujet ont néanmoins un trait caractéristique en commun: ils comportent un pronom anaphorique qui renvoie directement à leur cotexte gauche immédiat et met ainsi en évidence leur lien étroit avec ce cotexte.

(15) Mout volentiers et bonement / le vos randrai bien matinet, / foi que doi mon fil Martinet / et la moie fille Costance, / n'en soiez voz ja en doutance. (Roman de Renart, v. 9494-98)

(16) Se tu la reïne li ranz, / criens an tu avoir desenor? / De ce n'aies tu ja peor, / qu'il ne t'an puet blasmes venir ; / einz est pechiez del retenir / chose ou an n'a reison ne droit. (Chev. de la charrette, v. 3440-45)

(17) Mes tote voie ensi avint / que mes sire an cest chastel vint / ou il a deus filz de deable, / ne nel tenez vos mie a fable, / que de fame et de netun <démon> furent. (Chev. au lion, v. 5263-67)

Ainsi, ces trois $\mathrm{E}(\mathrm{imp})$ ne figurent pas en début d'intervention, et les pronoms anaphoriques en, ce et le y représentent respectivement un syntagme [(16)] ou une proposition entière [(15) et (17)] du cotexte gauche immédiat. De plus, ce cotexte gauche marque, tout comme le $\mathrm{E}(\mathrm{imp})$ négatif lui-même, la présence d'une relation coénonciative: il s'agit soit d'un énoncé déclaratif [(15) et (17)], soit d'un énoncé interrogatif à travers lequel le locuteur s'interroge sur l'état d'esprit de son allocutaire [(16)].

27 Il arrive cependant aussi que E(imp) comporte un pronom à valeur cataphorique, renvoyant à la suite immédiate de l'intervention du locuteur : 
Et je vos dirai voir prové, / si ne m'an mescreez vos pas, / que Gauvains tot eneslepas < sur-le-champ> / ne volsist pas qu'an l'esleüst / a roi,... (Chev. de la charrette, v. 6802-06)

Toutefois, ce $\mathrm{E}(\mathrm{imp})$ correspond à une incise qui s'insère dans un énoncé déclaratif, et le lien avec son cotexte gauche y est souligné par l'emploi de l'adverbe de phrase $s i^{13}$. Comme pour les autres occurrences, le cotexte du E(imp) de (18) révèle donc bien la présence d'un énonciateur et d'un co-énonciateur avant l'énonciation de cette injonction.

\section{E(imp) est lié à son cotexte droit : des emplois exceptionnels?}

Certains E(imp) de notre corpus se démarquent des occurrences traitées dans 1. et 2., dans la mesure où l'emploi du sujet de l'impératif paraît y être lié en premier lieu aux propriétés de son cotexte droit.

\section{A. Co-énonciation et emplois stylistiques du sujet de l'impératif}

Les deux coordinations suivantes en offrent un premier exemple :

(19)

Tu aime lui, e ele ame tei... (Mystère d'Adam, v. 13)

(20) Tien tu le tuen, et tu la toe. (Cligés, v. 2309)

31 Ici, E(imp) ne correspond pas au second, mais au premier élément d'une coordination contrairement à (1) et (6) - et la présence du pronom tu, anté- ou postposé à l'impératif, semble alors s'expliquer par des critères d'ordre stylistique. Tout comme l'emploi enclitique des pronoms régime de (19) ne s'impose pas d'un point de vue syntaxique ${ }^{14}$ mais sert surtout à mettre en valeur l'opposition entre les deux formes lui et tei, on peut penser que le sujet de l'impératif est employé ici pour créer un effet de contraste avec le pronom sujet de la seconde proposition coordonnée, en établissant une opposition tu / ele en (19) et celle entre deux $t^{15}$ renvoyant à des allocutaires différents - Soredamor et Alexandre - en (20). Cependant, nous ne considérons pas pour autant les E(imp) de (19) et (20) comme des exceptions à l'intérieur de notre corpus. Même si l'on constate, grâce à leur cotexte droit immédiat, une mise en relief des pronoms sujet dans ces deux occurrences, celles-ci ne s'avèrent pas pour autant incompatibles avec l'interprétation proposée pour les autres $\mathrm{E}(\mathrm{imp})$ traités jusqu'à présent : elles peuvent en effet également être rattachées à leur cotexte gauche; et celui-ci révèle, dans les deux cas, que E(imp) ne s'inscrit pas exclusivement dans la co-locution :

(19) Ce est ta femme e tun pareil : / Tu le devez estre ben fiël <fidèle>. / Tu aime lui, e ele ame tei... (Mystère d'Adam, v. 11-13) 
(20) Je t'abandon, / Alixandre, le cors t'amie ; / Bien sai qu'au cuer ne fauz tu mie <son cour ne te fait pas défaut>. / Qui qu'an face chiere ne groing <Quels que soient ceux qui fassent mauvaise figure ou grimace>, / L'un de vos deus a l'autre doing. / Tien tu le tuen, et tu la toe. (Cligés, v. 2304-09)

Ainsi, les deux E(imp) ne figurent pas en début d'intervention et leur cotexte gauche immédiat est déclaratif : dans (19), les vers 11 et 12 soulignent le bien-fondé de $\mathrm{E}(\mathrm{imp})$, et la locutrice de (20) marie ses deux allocutaires, Soredamor et Alexandre, en tenant compte de leurs inclinations, comme le suggère en particulier le vers 2306 de même que le cotexte plus large de l'énoncé :

Je vos os bien asseürer, / Se vos en avez boen corage <désir>, / J'asanblerai le mariage. (Cligés, v. 2270-72)

On observe donc bien la mise en place d'une relation de co-énonciation, par le locuteurénonciateur, avant la formulation de $\mathrm{E}(\mathrm{imp})$. Et c'est ce rapport co-énonciatif qui rend possible, comme dans les occurrences de 1. et 2., l'expression du sujet de l'impératif, ce qui permet alors, vu la construction symétrique de (19) et de (20), de créer un effet de contraste avec le deuxième pronom sujet contenu dans ces énoncés.

\section{B. Co-locution et co-énonciation ?}

Les occurrences suivantes représentent un cas de figure différent de celui illustré par (19) et (20) :

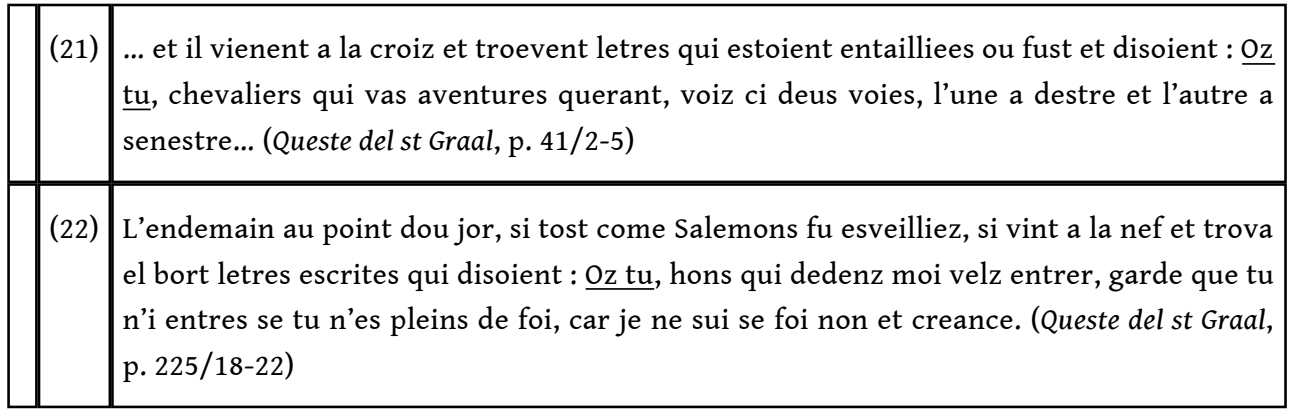

Dans la mesure où il s'agit d'inscriptions qui s'ouvrent sur la séquence « $\mathrm{Oz}$ tu $»^{16}, \mathrm{E}$ (imp) n'y comporte pas de cotexte gauche immédiat à travers lequel le locuteur-scripteur aurait pu établir une relation co-énonciative avec le destinataire du message. Comment alors expliquer la présence de ce pronom tu? Nous faisons l'hypothèse qu'il n'a pas, ici, la fonction syntaxique de sujet mais qu'il sert en revanche à interpeller le destinataire des énoncés qui suivent. Le cotexte droit de « $\mathrm{Oz}$ tu » représente un indice en faveur d'une telle interprétation: il correspond, dans les deux cas, à un appellatif qui contribue à l'identification de l'allocutaire, en soulignant, par l'emploi des propositions relatives, que l'inscription ne s'adresse pas à quiconque, mais seulement à un chevalier à la recherche d'aventures (21) ou à un homme souhaitant entrer dans la nef (22). De ce fait, le cotexte droit de « $\mathrm{Oz}$ tu » a pour fonction de préciser la relation entre le locuteur et l'allocutaire ou, plus exactement, entre le scripteur et le destinataire d'une inscription. Il nous parait alors possible d'attribuer au pronom tu un rôle comparable et de le considérer également 
comme un appellatif ${ }^{17}$. Nous rejoignons ainsi l'interprétation d'E. Baumgartner, qui propose de traduire ces deux passages de la manière suivante :

Chevalier, toi qui cherches les aventures, écoute :... (p. 53)

Ecoute, toi, qui que tu sois, qui veux monter à mon bord : Prends garde d'être plein

de foi car je suis la foi même. (p. 181)

Il est aussi possible de rapprocher nos occurrences de (23), où le verbe à l'impératif est également suivi directement d'un appellatif tu :

(23) Di, nains, di, tu qui le traïnes, / a quel forfet fu il trovez ? (Chev. de la charrette, v. 414-15)

De ce fait, (21) et (22) ne représentent pas non plus des exceptions à l'intérieur de notre corpus : l'emploi du pronom tu derrière la forme impérative n'y est nullement lié à la mise en place préalable d'une relation de co-énonciation, étant donné qu'il s'agit d'un appellatif, dont le rôle se situe par conséquent au plan co-locutoire ${ }^{18}$.

\section{Conclusion}

La prise en compte des concepts de co-locution et de co-énonciation dans notre analyse nous a permis, malgré la diversité des occurrences relevées, de trouver une propriété commune aux différents énoncés impératifs de notre corpus dans lesquels le sujet du verbe est exprimé. En effet, ces E(imp) ne s'inscrivent pas exclusivement dans la colocution: leur cotexte gauche et/ou l'emploi de la négation témoignent de la mise en place, avant la formulation de l'impératif, d'une relation de co-énonciation entre le locuteur-énonciateur et son allocutaire, qui devient alors co-énonciateur. En même temps, les données de notre corpus soulignent aussi deux autres traits caractérisant les injonctions à l'impératif de l'ancien français.

D'une part, la présence d'une relation co-énonciative dans le cotexte gauche de E(imp) n'entraîne pas obligatoirement celle d'un sujet tu/vos anté- ou postposé à l'impératif ; elle la rend seulement possible. C'est ce que montre notamment (9), où seul l'impératif perdone est accompagné de son sujet, ainsi que (24), qui peut être comparé à (1) :

(9) O Deus, vers rex, Jesu Crist, / ci <qui> tal don fais per ta mercit, / chi <qui> per huna <une $>$ confessïon / vide perdones al ladrun, / nos te laudam et noit et di, / de nos aies vera mercit; / tu nos perdone celz pecaz / q'e nos vetdest tua pietad. <les péchés que ta miséricorde verrait en nous>. (Passion, v. 301-08)

(24) Je vos desfi, et gardés vos... (Bel Inconnu, v. 403)

D'autre part, l'absence de relation co-énonciative dans le cotexte gauche de E(imp) n'exclut pas obligatoirement l'emploi d'un pronom tu/vos dans l'énoncé impératif. Mais, comme on le voit dans (21) et (22), ce pronom n'a alors pas le statut syntaxique de sujet il s'agit d'un appellatif, dont la fonction est d'interpeller l'allocutaire de l'énoncé. Tel est aussi le cas dans (25), où le locuteur emploie le pronom tu pour s'adresser à un deuxième allocutaire et pour définir ainsi une nouvelle relation co-locutoire : 
(25) Qu'est che, Cliquet? Est che bataille? / Laisse le [Pincedé] tost, et tu [Pincedé] lais lui [Cliquet]! <Lâche le vite, et toi, lâche Cliquet> (Jeu de st Nicolas, v. 923-24) ${ }^{19}$

En l'absence d'une morphologie discriminant le sujet tu et l'appellatif toi en ancien français, l'approche énonciative adoptée nous permet par conséquent de distinguer le sujet de l'impératif, dont l'expression est rendue possible par la co-énonciation, et l'appellatif, dont la fonction s'inscrit dans le cadre de la co-locution.

\section{BIBLIOGRAPHIE}

\section{Textes cités}

Aucassin et Nicolette, éd. J. Dufournet, Paris : Flammarion, GF, 1984.

La Chanson de Roland, texte établi d'après le manuscrit d'Oxford, éd. et trad. G. Moignet, Paris : Bordas, 1989.

Chrétien de Troyes : Les romans, éd. d'après la copie de Guiot. II : Cligés, éd. A. Micha, Paris : Champion, CFMA, 1975.

Chrétien de Troyes : Les romans, éd. d'après la copie de Guiot. III : Le chevalier de la charrette, éd. M. Roques, Paris : Champion, CFMA, 1970.

Chrétien de Troyes : Les romans, éd. d'après la copie de Guiot. IV : Le chevalier au lion (Yvain), éd. M. Roques, Paris : Champion, CFMA, 1963.

Chrétien de Troyes : Les romans, éd. d'après la copie de Guiot. V et VI : Le conte du Graal (Perceval), éd. F. Lecoy, V et VI, Paris : Champion, CFMA, 1979 et 1975.

Gormont et Isembart, fragment de chanson de geste du XIIe siècle, éd. A. Bayot, $3^{\text {ème }}$ éd. revue, Paris : Champion, CFMA, 1969.

Jean Renart : Galeran de Bretagne, roman du XIIIe siècle, éd. L. Foulet, Paris : Champion, (CFMA 37), 1975.

Jehan Bodel : Le jeu de saint Nicolas, éd. et trad. A. Henry, 3 ème éd. remaniée, Bruxelles : Académie royale de Belgique, (Mémoire de la classe des lettres LXV, 2) 1981.

Le Mystère d'Adam (Ordo representacionis Ade), éd. P. Aebischer, Genève : Droz et Paris : Minard, TLF, 1964.

La Passion. In : Avalle d'A. S., Cultura e lingua francese delle origini nella " Passion » di Clermont-Ferrand , Milan et Naples : Riccardo Ricciardi, 1962.

La Queste del saint Graal, roman du XIIIe siècle, éd. A. Pauphilet, Paris : Champion, CFMA, 1980.

La Quête du saint Graal, trad. E. Baumgartner, Paris : Champion, 1983.

Renaut de Beaujeu : Le Bel Inconnu, éd. G. P. Williams, Paris, Champion, 1991, CFMA. 
Le Roman de Renart, branches X-XI : Liétart, Renart et la mort de Brun, les vêpres de Tibert, éd. d'après le manuscrit de Cangé par M. Roques, Paris, Champion, 1958, CFMA.

Villehardouin : La conquête de Constantinople, éd. et trad. E. Faral, Paris, Société d'Edition Les Belles Lettres, 1973.

\section{Bibliographie}

BRUNOT F., BRUNEAU Ch. (1969) Précis de grammaire historique de la langue française, Paris : Masson, rééd.

BURIDANT, Cl. (2000) Grammaire nouvelle de l'ancien français, Paris : SEDES.

DANON-BOILEAU L., MOREL M.-A., PERRIN I. (1992) «L'impératif en français et en anglais contemporains », Journal of Linguistics 9, Aarhus (Danemark), 157-180.

DUCROT, O. et al. (1980) « Mais occupe-toi d'Amélie ». In : Les mots du discours, Paris, Editions de Minuit, (Le Sens commun), 93-130.

FOULET, L. (1990) Petite syntaxe de l'ancien français, Paris : Champion, rééd., CFMA [1 ère éd. 1919].

HASENOHR, G. (1990) Introduction à l'ancien français de Guy Raynaud de Lage, Paris : SEDES.

LE GOFFIC, P. (1993) Grammaire de la phrase française, Paris : Hachette.

MARCHELLO-NIZIA, Ch. (1985) Dire le vrai : l'adverbe " si » en français médiéval. Essai de linguistique historique, Genève : Droz

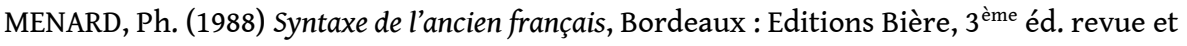
augmentée.

OPPERMANN, E. (1992) «Les impératifs du verbe savoir en moyen français », Travaux de linguistique 25, 127-36.

OPPERMANN, E. (2001) «Or te dirrai, e tu m'ascoute : quelques remarques concernant l'expression du sujet de l'impératif en ancien français ", Le français et ses usages à l'écrit et à l'oral. Dans le sillage de S. Lafage, Paris : PSN, 245-52.

PERRET, M. (1994) L'énonciation en grammaire du texte, Paris : Nathan (128).

ROULET, E. (1981) «Echanges, interventions et actes de langage dans la structure de la conversation », Etudes de linguistique appliquée 44, 7-39.

ROULET, E. (1986) «Complétude interactive et mouvements discursifs », Cahiers de linguistique française 7 (Genève), 189-206.

\section{NOTES}

1. Les auteurs de cet article parlent de co-locution «chaque fois qu'il s'agira de désigner la situation de co-présence des interlocuteurs » (p. 157); le terme de co-énonciation fait référence à la prise en charge du contenu de l'énoncé, de l'opinion exprimée ou de l'événement posé, par le locuteur (énonciateur) et l'allocutaire (co-énonciateur). Il implique la prise en compte, par le locuteur-énonciateur, de la pensée qu'il prête à son co-énonciateur.

2. Nous distinguons ce type d'occurrences de celles où tu/vos correspond à un appellatif - emploi qui est toujours possible en français contemporain (cf. Toi, viens ici !). Sur la question $\mathrm{du} t u / v o s$ appellatif, cf. 3.B.

3. En effet, les manuels d'ancien français avancent en principe les mêmes explications pour rendre compte de ces deux types d'énoncés impératifs. Ainsi, Cl. Buridant classe, dans sa 
Grammaire nouvelle de l'ancien français, les impératifs renforcés "par le pronom » (sujet) et ceux qui sont renforcés « par les adverbes antécédents » sous la même rubrique (2000, § 624). Cf. aussi Brunot \& Bruneau (1969 : 323).

4. Nous entendons par « cotexte » le contexte linguistique d'une occurrence.

5. Nous excluons cependant de cette étude les occurrences de «Vos soiez li bien venuz / Bien soiez vos venuz » (qui correspondent à un souhait de bienvenue relativement figé), ainsi que les impératifs de savoir («Vos sachiez / Ce sachiez vos» <que P>) qui sont directement liés à leur cotexte déclaratif. Sur ce dernier point, cf. notre article (1992).

6. Ce corpus est constitué de 25 textes en vers et en prose datant essentiellement des XII ${ }^{\mathrm{e}}$ et XIII ${ }^{\mathrm{e}}$ siècles, mais incluant aussi la Vie de saint Alexis ( $\mathrm{XI}^{\mathrm{e}} \mathrm{s}$.) et la Passion du Christ ( $\mathrm{X}^{\mathrm{e}} \mathrm{s}$.). Les références des textes cités sont données à la fin de l'article.

7. Pour une analyse détaillée des coordinations du type «E1 - ET - E(imp)», cf. notre article (2001).

8. «la conjonction et, jetée en tête de la principale, joue le rôle d'un adverbe de reprise " (Ménard, 1988, § 195).

9. Selon P. Le Goffic (1993), l'appellatif peut avoir deux fonctions : l'identification de l'allocutaire et/ou sa caractérisation (qualification). La mention de qualités de l'allocutaire peut alors aussi contribuer à l'identification de celui-ci, « en levant par une propriété discriminante l'ambiguïté situationnelle possible de tu ou vous (...) : Eh, toi, le petit, viens ici!» (p. 374).

10. Selon E. Roulet (1981 et 1986), un échange verbal de base entre deux interlocuteurs est composé de trois interventions: une intervention initiative (par ex. une injonction), une intervention réactive (il s'agit de la réponse à l'intervention initiative) et une intervention évaluative (par ex. des remerciements).

11. Nous nous appuyons ici sur l'analyse de O. Ducrot: «Tout acte de parole est compris comme comportant des prétentions; prétentions d'une part à être légitime, à avoir le droit d'être accompli, bref à être autorisé, et d'autre part à faire autorité, c'est-à-dire à infléchir les opinions ou les comportements verbaux ou non verbaux du destinataire (...). Quand on s'oppose à un acte de parole en lui objectant mais $Q$, on s'oppose à de telles prétentions. » (Ducrot et al., 1980, p. 126-27).

12. Distinction qui ne s'imposait pas dans 1 ., puisque la présence de l'adverbe de négation entraîne forcément la postposition du pronom sujet derrière le verbe.

13. Dans la mesure où un énoncé introduit par si est généralement fondé sur son cotexte gauche immédiat: celui-ci devient alors un "préconstruit » légitimant l'énonciation de la suite de l'intervention ; cf. C. Marchello-Nizia (1985).

14. Étant donné que la présence des pronoms sujet tu et ele devrait normalement s'accompagner de l'emploi de pronoms régime proclitiques.

15. Même si ces deux tu n'ont pas le même statut syntaxique, cf. note 19.

16. Nous n'avons relevé ce type de E(imp) que dans la Queste del saint Graal.

17. Cette interprétation entraînerait, dans l'édition, l'introduction d'une virgule entre l'impératif oz et le pronom $t u$.

18. Bien que l'énonciation écrite soit par définition "différée » (Perret, 1994) et exclue par conséquent la co-présence des interlocuteurs dans une situation d'énonciation unique, le locuteur s'adresse, par le biais des énoncés impératifs et des appellatifs, directement à son allocutaire, comme si celui-ci était présent au moment de l'énonciation. Cet aspect est souligné, dans nos exemples, par l'emploi de l'impératif du verbe « oïr ».

19. Nous analysons de la même manière le deuxième tu de (20): il sert à interpeller un deuxième allocutaire, Alexandre, alors que le premier tu, sujet de l'impératif tien, fait référence à Soredamor. Cf. 3.A. 


\section{AUTEUR}

\section{EVELYNE OPPERMANN}

Université Paris III 